\title{
DEEP CONVOLUTIONAL NEURAL NETWORKS FOR DEGRADED PRINTED KANNADA CHARACTER RECOGNITION
}

\author{
Sridevi T.N \\ Research scholar \\ Department of studies in Computer Science, \\ University of Mysore, ManasaGangothri Campus, \\ Mysuru,Karnataka,India \\ tn.sridevi1@gmail.com \\ LalithaRangarajan \\ Professor \\ Department of studies in Computer Science, \\ University of Mysore, ManasaGangothri Campus, \\ Mysuru, Karnataka, India \\ lali85arun@yahoo.co.in
}

\begin{abstract}
Recognition of degraded printed Kannada characters is a challenging research problem. Proposed in this paper is a deep convolutional neural network for recognition of degraded printed Kannada characters. Characters in some old Kannada texts are affected by various degradations that result in breakages and dilations of characters introducing challenges in the process of recognition. The architecture consists of three levels, the first two levels with ReLu, Max pooling layers, and the third level with just ReLu. The output of these is input to fully connected layer which performs classification of characters. Experimental analysis is carried out using 156 classes of characters each class with 100 instances. Performance is evaluated for 4 epochs with 60 iterations per epoch. Highest classification accuracy of $99.51 \%$ has been reported for $75 \%$ training.
\end{abstract}

Keywords:Deep learning, Degraded character recognition, Kannada printed characters, Classification

\section{Introduction}

In the recent days from literature, it is understood that deep learning models to carry out complex computer vision and pattern recognition applications have been highly successful. Kannada character set has large number of classes owing to the presence of high number of consonants and vowel modifiers. Although the machine learning models provide promising results for few of the complex recognition task such as character recognition, deep learning framework for Kannada character recognition is expected to outperform the conventional machine learning models as these networks have been shown to handle multi features and multi class problems much more efficiently. Hence our attempt of deep CNN for degraded Kannada character recognition.

The existence of higher degree of modifiers in a character set leads to formation of huge number of complex compound characters,[1]. A complex compound character is a combination of a base character and two or more vowel and consonant modifiers [2]. Recognition of the characters in south Indian scripts like Kannada is always

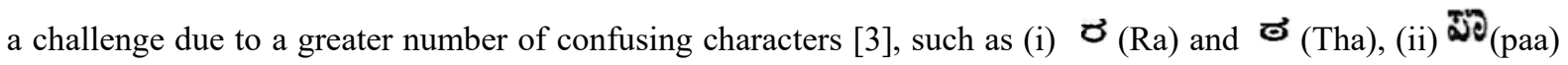
and (Shaa). A couple of attempts by various researchers since 1960's is discussed here. The works reported so far are characterized into printed and handwritten character recognition systems. The proposed work is towards printed character recognition for Kannada. The major challenges in the proposed work include the degradations associated with the characters of various printed fonts.

Recognition of degraded printed characters is comparatively a challenging problem. The deformations within the characters are usually affected due to aging of the document images. Various techniques for degraded kannada characters by Sandhya .N et al [4]. The model uses a single layer neural network and achieved an accuracy of $98.9 \%$ for the datasets consists of synthetically generated broken characters. 
The proposed research work by N. Shobha Rani et al [5] can handle recognition of degraded character images and handwritten character images. The training of the degraded characters by deep convolution neural networks called Alex net. And synthetically generated handwritten samples are collected from the users belong to the age group between 18 to 30. Experimentation conducted on the printed datasets includes vowels, consonants, compound characters. Accuracy of $91.3 \%$ and $92 \%$ is reportedfor printed and handwritten character samples respectively.

Sandhya. $\mathrm{N}$ et al [6] have proposed a model wherein degradation in characters are measured in scales called high, medium, low. A new method FDA has been employed to compare recognition accuracy with other approaches namely SVM and FLD.The characters have been rebuilt to improve recognition accuracy. The authors have conducted extensive experiments and their model of neural network has achievedbetter recognition accuracy.

A stepwise framework proposed by Sandhya .Net al [7] for recognition of degraded Kannada characters as follows: Pre-processing, rebuilding of character components, followed by feature extraction and classification. An accuracy of $99 \%$ has been observed.

The model devised by V. N ManjunathAradhya et al [8] for recognizing Kannada characters using FLD Analysis. The model explores the differences between the multivariate classes. System tested on noisy characters from both Kannada and English. The model achieved 96.1\% of accuracy.

A framework called KannadaRes-NeXthas been proposed by Saini et al [9] for Kannada numeral recognition. Res-NeXthas been used to classify the images.Kannada-MNIST trainingand testing dataset has 60000 and 10000 numerical images repectively. Dig-MNIST dataset acts as out-of-domain test sample set with 10,240 images. Two more test images of AugKannada-MNIST and AugDig-MNIST with 10,000 and 10,240 have also been introduced. Observed accuracies as follows: Kannada-MNISTwith 97.36\%,Dig-MNIST with $79.06 \%$,augmented maintest set and augmented Dig-MNIST with $96.32 \%$ and $77.31 \%$ respectively.

Chandrakala H. T and Thippeswamy G [10] deployed Deep convolution neural network for the recognition of historical Kannada handwritten characters. Experiments carried out on digitized E-stampages using SVM classifier and an accuracy of $70 \%$ is achieved.

In a later work by Thippeswamy G, and Chandrakala H. T [11], local binary pattern features are employed for historical handwritten Kannada character recognition. However, the features employed are not efficient towards handwritten character recognition. LBP features are extracted on the segmented character images. Decision tree classifier resulted in improved performance.

H.R. Shiva kumar and A.G. Ramakrishnan [12] proposed a technique called LipiGnani for any language document printed in Kannada scripts using machine learning strategy that uses red-black trees, symbolic segmentation and decision tree based classification.Test databases consists of scanned images of Kannada, Sanskrit, Konkani and Tulu language books. Experimentation is conducted on around 35 books of non-degraded printed text. An accuracy of LipiGnaniover Google's Tesseract OCR is $4 \%$ more on Kannada, $8 \%$ more on Tulu and Sanskrit, $25 \%$ more on Konkani dataset.

G. S. Monisha and S. Malathi [13] have presented a survey on variety of techniques that can be used for handwritten character recognition suitable for unconstrained document development using pattern recognition techniques. Difficulty is faced in recognition of stroke edges present in handwritten character.

V. Sathya Narayanan and N. Kasthuri [14] proposed a technique for English character recognition, using the following steps:binarization for noise removal, LBP for extracting features, SVM for classification. However, the experiments are conducted only on printed English fonts of selected typed and have reported accuracy of $94.6 \%$.

C.V Aravinda et al [15] employed model based and detailed extraction of statistical features followed by distance classifier to recognize south Indian printed characters of non-noisy types.

Research by Imran et al [16] adapted connected component analysis and template matching technique for recognition of printed distorted English characters. It is observed from various research that template matching techniques are sensitive to handling of variable pixel relationships associated with the same class type. The proposed DCR has been shown to have an accuracy of $97 \%$ over datasets.

A method by Parashuram Bannigidad and Chandrashekar Gudada [17] for reconstruction, digitization, and Recognition of historical Kannada handwritten characters have used HOG feature descriptors followed by KNN and SVM classifiers for recognition. An accuracy of $92.3 \%$ and $96.7 \%$ is observed. 
An adaptive thresholding technique has been applied by H. R. Shiva Kumar, and A. G. Ramakrishnan [18]on gray scale word images for nonlinear enhancement.The word images are collected across ancient documents to connect the broken strokes of characters. Experiments are conducted over 1685 degraded sample images collected from ancient Kannada documents. They found an increased recognition accuracy of $14.8 \%$ and 5.6\% over Unicode using SVM classifier and Google's Tesseract OCR respectively.

Kavya Ramalingam and Ramamurthy Bhojan [19] proposed a technique for broken character recognition from degraded documents. Lines are separated using projection profiles, characters are identified using chain coding,broken characters are found using mean based thresholding. However, chain codes are most sensitive data representation techniques to handle degradation in broken characters. An accuracy of $92.88 \%$ is observed both on color image documents and also on black and white image.

Chandrashekar Gudada and Parashuram Bannigidada [20] proposed technique for historical Kannada handwritten character recognition using techniques like bounding box segmentation, geometrical features and K-Nearest Neighbour classifier. The accuracy of the system is found to be $97.83 \%, 97.78 \%, 97.92 \%$, $97.87 \%, 100 \%, 97.87 \%$ and $97.96 \%$ is evaluated towards Kadamba, BadamiChalukya, KalyanaChalukya, Hoysala, Vijayanagara, Mysore Wodeyars, and Aadhunika Kannada. It is observed that most of the handwritten scripts evaluated are developed in constrained environments.

B Gangamma and Srikanta Murthy K [21] have proposed a work where the focus is on enhancement of ancient Kannada document images of palm scripts. Adaptive Histogram equalization and grayscale morphological operations with spatial filters are used to perform the enhancement. Blurred and skewed images are considered as the limitations of their work.

Ranganatha D and Ganga Holi [22] have worked on an enhancement technique for degraded document images using adaptive bilateral filtering techniques. Method is found to work well for palm leaf manuscript and degraded images.

Munish Kumar et al [23] have devised a model for recognition of handwritten Gurumukhi characters using diagonal and transition features using KNN classifier. These features are calculated by distributing the points over the bitmap image of character. Around 3500 Gurmukhi characters have been considered for experimentation. Model achieves an accuracy of $94.12 \%$ for diagonal features and KNN classifier.

Attempts were made by Karthik S et al [24] to remove the noise present in Kannada characters using Run length count. The datasets considered for experimentation are epigraphical and images of printed document with noise.

Antony P.J et al [25] have employed Haar features in combination with Adaboost algorithm for recognizing handwritten Tulu script. Finally recognized characters are mapped into corresponding Kannada characters to make it readable by digital technology for next generation.

Sandhya, $\mathrm{N}$ et al [26] have proposed a new approach to rebuild the broken Kannada characters. Characters are segmented using region-based segmentation.End point algorithm has been used to rebuild the characters. Experiments are conducted over a dataset of 100 character samples. The recognition accuracy is tested before and after rebuilding a character sample. It is observed that recognition rate has improved to $89 \%$ after rebuilding the sample when compared to $53 \%$ without rebuilding.

Sridevi T N, LalithaRangarajan [27] proposed a technique for removal of background noise present in documents extracted from old Kannada documents. Enhancement of the documents consists of series of steps as follows: Contrast enhancement, Filtering, Binarization followed by BIA technique. Around 175 documents with high degradations are scanned for experimentation and 25 datasets collected from DIBCO database. The method has resulted in an appreciable accuracy towards character retention for highly degraded ancient documents. Obtained accuracy has been compared to widely used techniques Sauvola, Otsu, Gaussian.

Based on the review of research on character recognition systems, the following observations are made.

1. Numerous works are reported in the area of de-noised printed character recognition systems however these techniques are suitable for Non-south Indian scripts based character recognition.

2. In a couple of works the techniques such as chain codes, geometrical and statistical distance features are highly used for recognition of degraded handwritten characters, which are sensitive towards noise or any other degradation in a character instances,thus, making it not suitable to handle challenges in degraded characters.

3. It is also evident that most of the works focus on enhancement of the degraded document images rather than recognition. 
Organization of the rest of the paper is as follows: Working of proposed methodology for recognition of degraded and printed Kannada characters are described in Section 2. Results of the proposed technique are presented in Section 3. Final conclusions and work planned for future are briefed in Section 4.

\section{Proposed Methodology}

The model for recognition of degraded printed Kannada characters is devised using a deep Convolutional Neural Network (CNN). Figure 1 shows the architecture of CNN employed for degraded and printed Kannada character recognition.

The framework consists of an input layer followed by three level convolution learnable layers followed by fully connected classification layer.

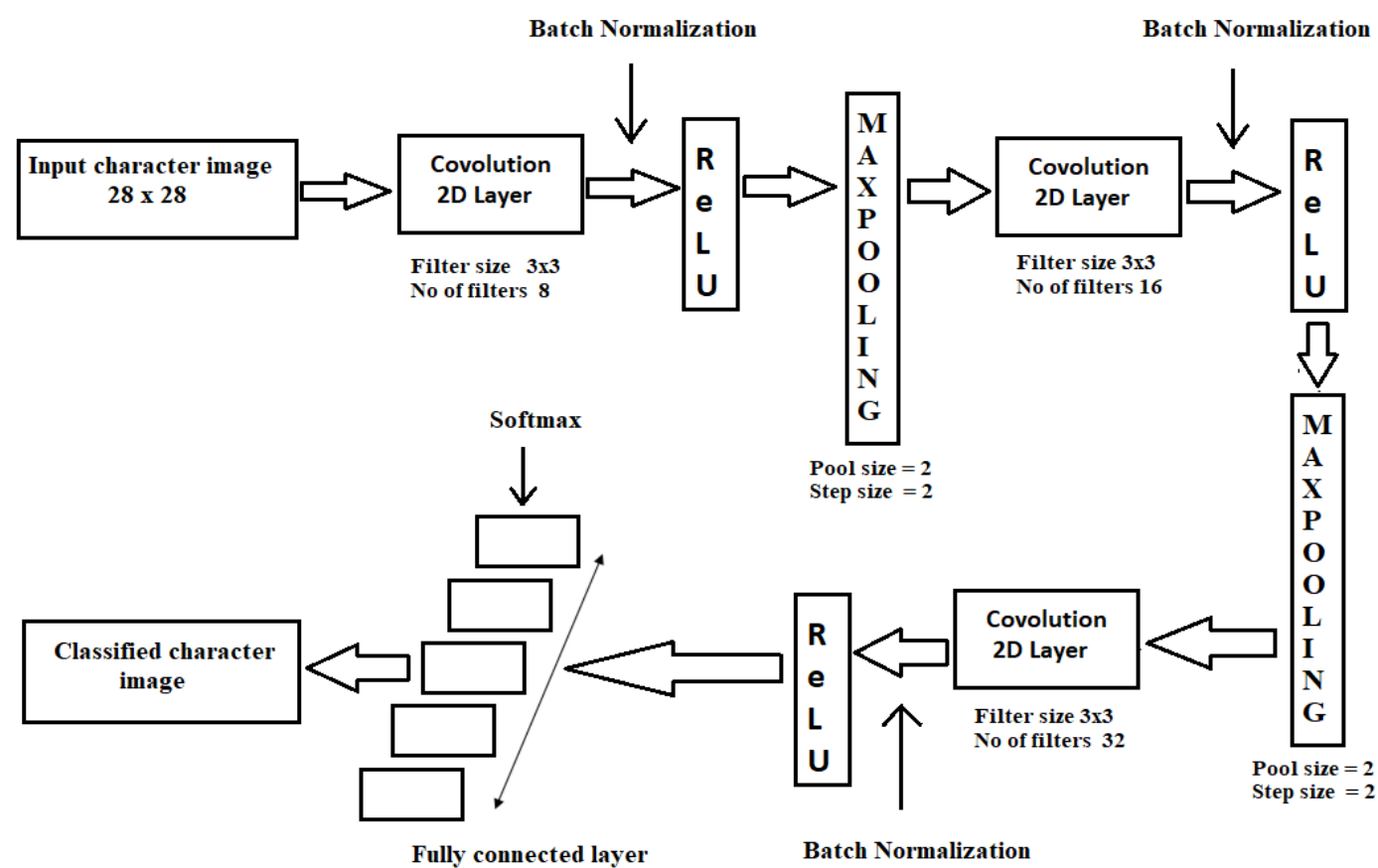

Figure 1: Architecture of deep convolution neural network for degraded printed Kannada character recognition

\subsection{Input layer}

The layer is responsible for acquisition of a 2dimensional array of numbers that corresponds to the pixels of an input image. For example, the input image of dimensions $28 \times 28$ of 784 pixels will be fed as input as it is for convolutions. Figure 2 shows a degraded character, the pre processed character and the input binary matrix.

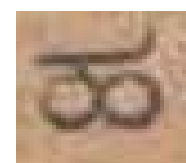

(a) Sample degraded dataset

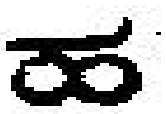

(b) Pre-processed sample

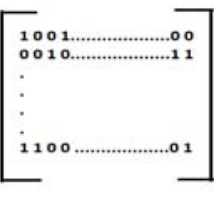

(c) $28 \times 28$ Input matrix

Figure 2: Sample character extracted

\subsection{Convolution layer}

Convolution is the process of representing the data from lower level raw data to higher level in abstract form. In this layer of deep learning model, a set of predefined convolution filters of fixed dimensions are employed to detect and interpret the patterns pertaining to an image to include edge, texture details with respect to various regions of the objects. The process results in composition of features required to detect and classify the complete object in an image. 
In the proposed methodology 3 levels of convolution layers are introduced. In the level 1 the convolution is carried out with the filter size $3 \times 3$ with the help of 8 filters. In the level 2 a filter size $3 \times 3$ is used with increment in the number of filters to 16.Further the number of filters in level 3 is raised to 32 with a constant filter size of $3 \times 3$. Deeper understanding of the features leading to global abstract representation of the image is possible by increasing the number of filters as we move down the layers. Additionally, increasing the number of layerswill also help in the extraction of high-level features adequate to represent the geometric structure along with deformations of the character. It is observed from various deep learning architectures padding the input image by adding rows and columns results in much wider network. The wider networks have been shown to learn better. In this research the images are degraded characters of dimensions $28 \times 28$ which are padded and a threelevel deep learning process employed to achieve higher recognition rates.

Table 1 shows the details of input parameters used in convolution layers.

Table 1: Details of filters used for feature extraction

\begin{tabular}{|l|l|l|}
\hline \multirow{2}{*}{ Convolution layer } & \multicolumn{2}{|l|}{ Input specifications } \\
\cline { 2 - 3 } & Filter size & No. of filters: \\
\hline Level 1 & $3 \times 3$ & 8 \\
\hline Level 2 & $3 \times 3$ & 16 \\
\hline Level 3 & $3 \times 3$ & 32 \\
\hline
\end{tabular}

Batch normalization layers between convolutional layers and nonlinearities such as ReLu layers has dual advantage. It speeds up training, reduces sensitivity to network initialization, activation function is more viable and simplifies creation of deeper networks.

\section{$2.3 \operatorname{ReLU}$}

An activation function is usually employed to identify the responses for selection of an appropriate neuron in a particular layer. Rectified Linear Unit (ReLU) acts as a non-linear activation function while the data is switched from one layer to another layer. ReLu selects a neuron with maximum function probability. ReLU is an element wise operation applied for each pixel and replaces all negative pixel values in the feature map by zero and output of ReLu is max(Zero,Input).

\subsection{Max pooling}

In general, pooling is the process of reducing the dimensionality of the image to achieve feature abstraction. Pooling is a process that controls the over fitting of the data, thereby reducing the recognition error. Deep learning architecture that are adopted for classification of the image data requires huge amount of training time and hence performing maxpooling reduces the huge image data of higher dimensions to lower dimensional problems. For example, applying maxpooling with a stride length of 2reduces every $2 \times 2$ region in an image to one value (which is maximum of all pixel intensities in the $2 \times 2$ region). Additionally pooling will also helps in retention of rotational and position invariant features in an image. The pooling process will finally result in extraction of dominant feature values from the given image.

\subsection{Fully connected layer}

The inputs to the fully connected layer considered as the features value outcomes obtained after applying the 3 levels of convolution. This is a learning layer as in a conventional neural network. In the instance of error propagation in convolution neural network, output layer back propagates to initiate re-learning from fully connected layer.

\section{Experimental Analysis}

In the proposed research, experimentation is conductedon a data set of 156 degraded characters with each class consisting of about 100 instances. Multiple sets of experiments conducted with various training testing ratios. Architecture of network employed for recognition of characters is constant in all experimental set up. The data set includes some skew in all characters.

Experimentations are conducted with the proposed deep learning model in 4 epochs and up to 100 iterations in each epoch for both experiments. The results prove that the convergence of training data with validation data is stabilized starting from epoch 2 onwards in both the experiments. Figure 3 shows the results of training \& 
validation data which is a plot of accuracy and figure 4 is plot of loss in the case of $75 \%$ training. It is evident from figures 3 and 4 that in about 350 iterations accuracy is maximum and loss is minimum.

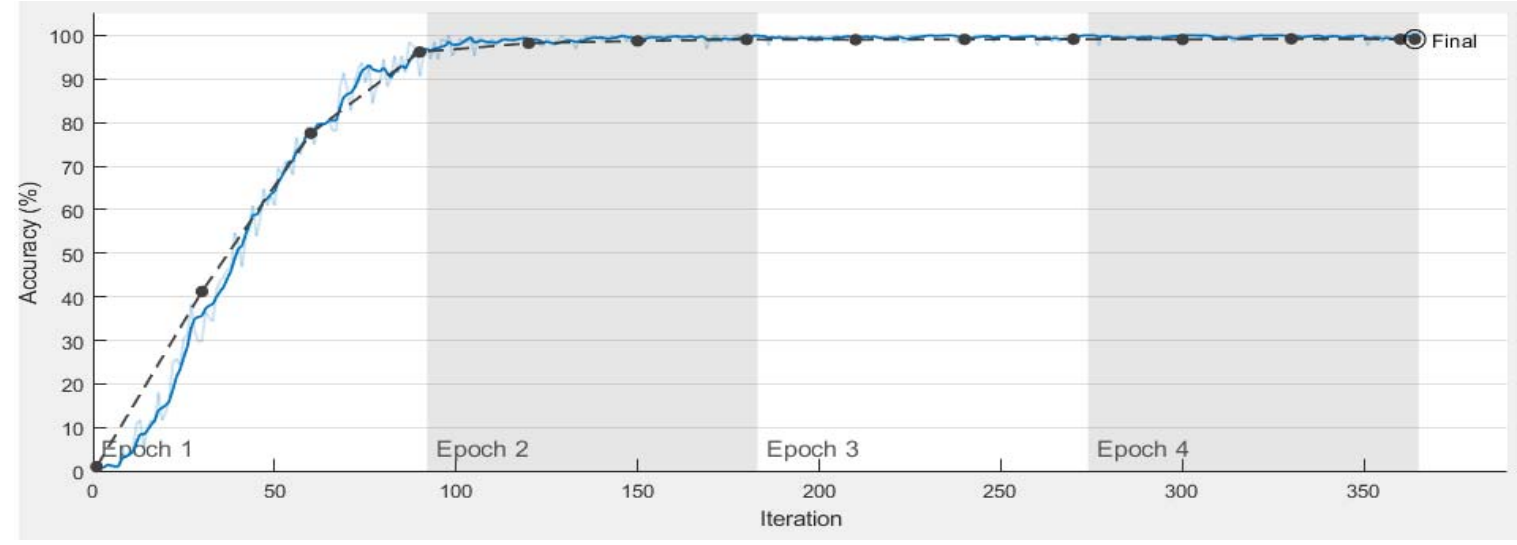

Figure 3: CNN performance - No. of iterations vs. Accuracy- $75 \%$ to $25 \%$

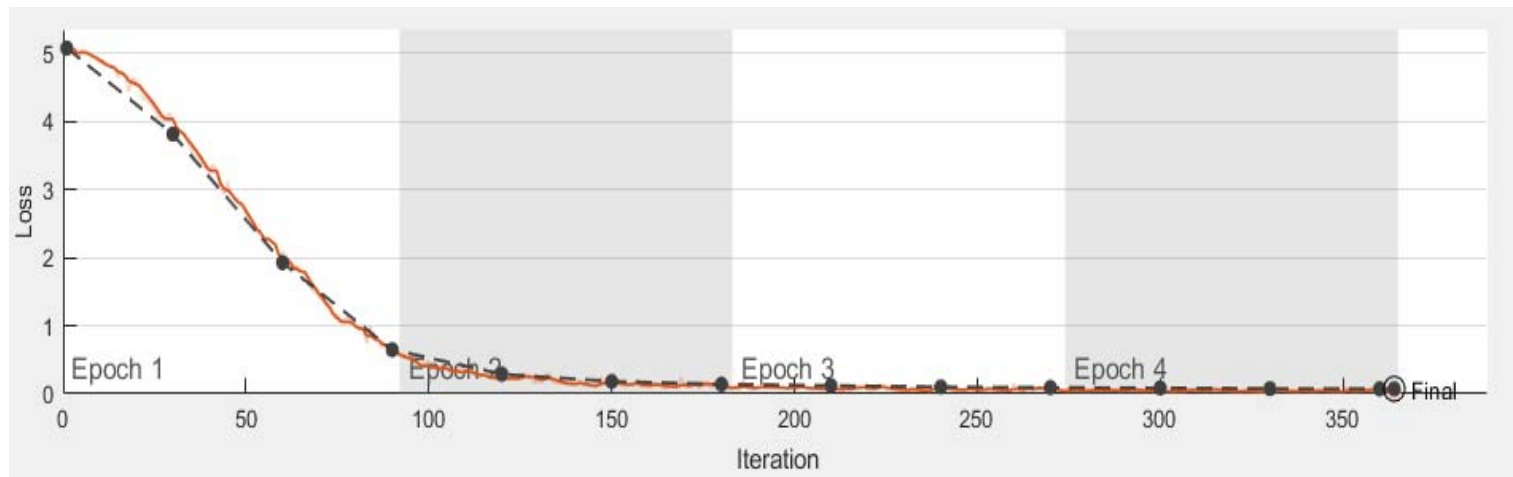

Figure 4: CNN performance - No. of iterations vs. Loss- $75 \%$ to $25 \%$

Figure 5 shows the results of accuracy of training \& validation data over iterations. Figure 6 is a plot of loss (recognition failures) with respect to number of epochs for $50 \%$ training. It is to be noted that in about 240 iterations accuracy or loss stabilizes. It is easy to infer that $75 \%$ of the data for training yields the best result. The learning model uses the cross-entropy loss function to optimize the error rateand to achieve more accurate predictions.

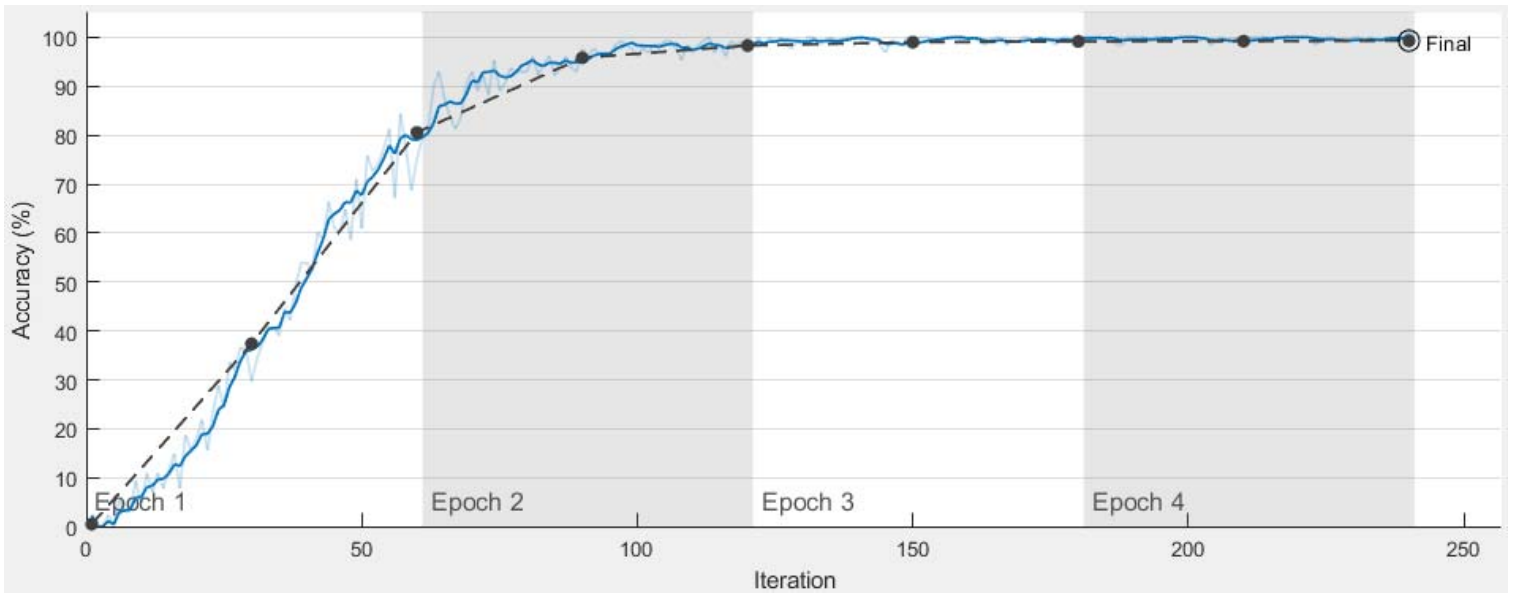

Figure 5: CNN performance - No. of iterations vs. Accuracy- $50 \%$ to $50 \%$ 


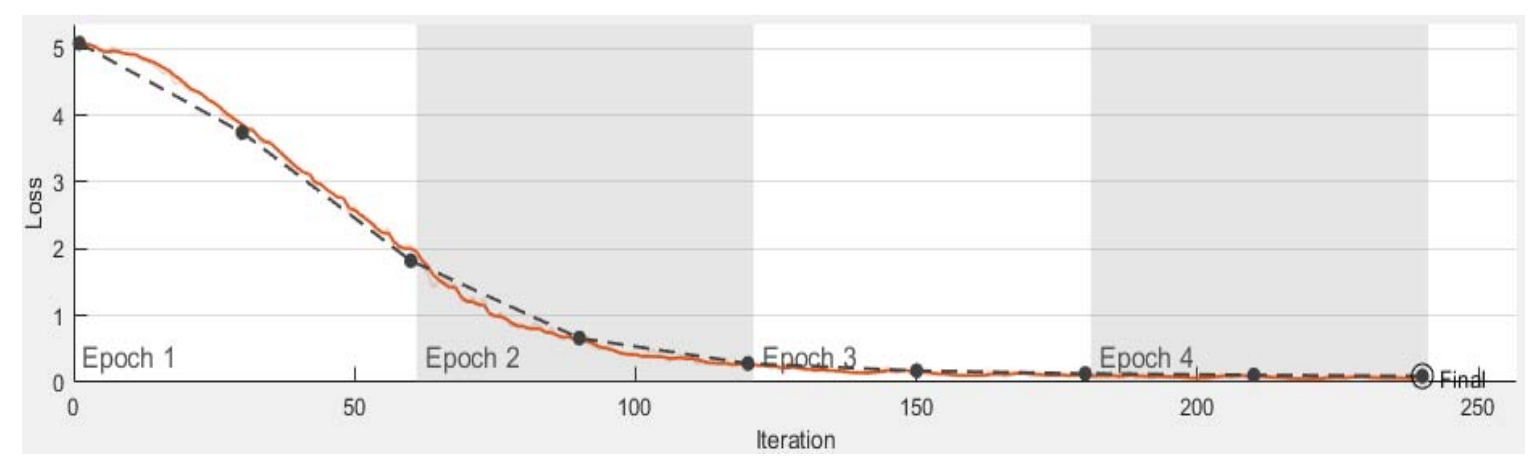

Figure 6: CNN performance - No. of iterations vs. Accuracy-50\% to $50 \%$

From the figures 3 to 6 , it is evident that the results are promising towards recognition of degraded printed Kannada characters. It is also noticed that the convergence of training to validation data is seen clearly from epoch 2 in both the experiments. Thus, Convolutional neural networks are proved to be robust towards recognition of degraded printed character recognition.

Table 2 shows the training and test samples considered for experimentation. From table 2, it is observed that the deep $\mathrm{CNN}$ has yielded highest accuracy when trained with $75 \%$ of the data.

Table 2: Training sample details

\begin{tabular}{|l|l|l|}
\hline SI No & Train to test & Accuracy \\
\hline 1 & $30 \%-70 \%$ & $97.85 \%$ \\
\hline 2 & $40 \%-60 \%$ & $99.00 \%$ \\
\hline 3 & $50 \%-50 \%$ & $99.24 \%$ \\
\hline 4 & $75 \%-25 \%$ & $99.51 \%$ \\
\hline 5 & $90 \%-10 \%$ & $99.36 \%$ \\
\hline
\end{tabular}

Table 3 highlights the recognition accuracy achieved on degraded documents. The comparative analysis between the existing and proposed techniques have been recorded in table 3. It is clear from the projected experimental results in the table 3 that the recognition accuracy of the proposed method is higher than the other existing methods. Our data set are characters extracted from old degraded printed Kannada documents which are subjected to preprocessing using Binary Image Analysis(BIA) technique [27].

Table 3: Comparative analysis of degraded printed Kannada character recognition

\begin{tabular}{|c|c|c|c|c|}
\hline SI No & Ref No & Total datasets experimented & Techniques & Accuracy \\
\hline 1 & 4 & $\begin{array}{l}2450 \text { broken character dataset synthetically } \\
\text { generated }\end{array}$ & $\mathrm{NN}$ & $98.9 \%$ \\
\hline 2 & 6 & $\begin{array}{l}250 \text { real datasets from historical, } 150 \times 49=7350 \\
\text { Synthetically generated dataset }\end{array}$ & FDA(Fit Discriminant Analysis) & $99.38 \%$ \\
\hline 3 & 8 & $\begin{array}{l}21560 \text { both clear and degraded characters(Kannada } \\
\text { and English) [Kailasam, Kasturi,Times new } \\
\text { Roman,Arial] }\end{array}$ & $\begin{array}{l}\text { FLD(Fisher Linear Discriminant } \\
\text { Analysis) }\end{array}$ & $98.2 \%$ \\
\hline 4 & 26 & 100 degraded Kannada characters & End point algorithm & $89 \%$ \\
\hline 5 & Proposed Method & $\begin{array}{l}15600 \text { degraded Kannada characters extracted from } \\
\text { old documents }\end{array}$ & Deep Convolutional Neural Network & $99.51 \%$ \\
\hline
\end{tabular}

Table 4 shows the classification accuracy achieved with traditional classifiers versusdeep CNN.In our earlier work " Degraded Character Recognition from Old Kannada Documents " Features are extracted using HOG and classified using Fine Gaussian SVM, Fine KNN, Medium KNN, WKNN, Cosine KNN, Cubic KNN, Ensemble Adaboost classifier with $94.6 \%, 98.5 \%, 90.9 \%, 97.9 \%, 92.1 \%, 90.4 \%, 32.5 \%$ respectively for a cell(block) size of $4 \times 4$ and $94.9 \%, 97.7 \%, 89.1 \%, 96.7 \%, 89.1 \%, 88.5 \%, 12.0 \%$ respectively for a cell(block) size of $8 \times 8$. Performance of Fine KNN is found to be the best of all classifiers experimented with and this accuracy is taken for comparison in this table. The proposed Deep CNN for feature extraction and classification has yielded an accuracy of $99.51 \%$. Table 4 also recorded an accuracy of $3.3 \%$ when tested across 30 samples in current available online OCR ((https://www.newocr.com/) $)$ 
Table 4: Classification accuracy

\begin{tabular}{|l|l|l|l|l|}
\hline $\begin{array}{l}\text { SI } \\
\text { No }\end{array}$ & Feature extraction type & Size of the dataset & $\begin{array}{l}\text { Classification } \\
\text { Technique }\end{array}$ & Accuracy \\
\hline 1 & HOG & 10440 & Fine KNN & $98.5 \%$ \\
\cline { 5 - 6 } & & $4 \times 4$ & & $97.7 \%$ \\
\hline 2 & Proposed Method & 15600 & Deep CNN & $99.51 \%$ \\
\hline 3 & Reference [28] & 30 Samples & Online OCR & $3.3 \%$ \\
\hline
\end{tabular}

\section{Conclusion}

In a nutshell, an efficient deep learning model using convolution neural networks is proposed in this research. An attempt is made towards recognition of degraded printed Kannada characters collected from old Kannada documents. 156 degraded characters and 100 instances (covering same character from different documents as well as skewed characters) of each character is the data set on which experiments are conducted. It is observed that, three level deep convolution layers comprising of ReLu and max pooling steps results in about $99.51 \%$ of accuracy towards recognition of degraded Kannada characters.

\section{References}

[1] Pal, U., Wakabayashi, T., \& Kimura, F. (2007, December): Handwritten Bangla compound character recognition using gradient feature.In 10th international conference on information technology (ICIT 2007) (pp. 208-213).IEEE.

[2] Das, N., Das, B., Sarkar, R., Basu, S., Kundu, M., \&Nasipuri, M. (2010): Handwritten Bangla basic and compound character recognition using MLP and SVM classifier.arXiv preprint arXiv:1002.4040.

[3] Suen, C. Y., Guo, J., \& Li, Z. C. (1994): Analysis and recognition of alphanumeric handprints by parts.IEEE transactions on systems, man, and cybernetics, 24(4), 614-631.

[4] Sandhya, N., \& Krishnan, R. (2016, March): Broken Kannada character recognition-a neural network based approach. In 2016 International Conference on Electrical, Electronics, and Optimization Techniques (ICEEOT) (pp. 2047-2050). IEEE.

[5] Rani, N. S., Chandan, N., Jain, A. S., \& Kiran, H. R. (2018): Deformed character recognition using convolutional neural networks. International Journal of Engineering \& Technology, 7(3), 1599-1604.

[6] Sandhya, N., Krishnan, R., Babu, D. R., \& Rao, N. B. (2019): An efficient approach for handling degradation in character recognition. International Journal of Advanced Intelligence Paradigms, 14(1-2), 14-29.

[7] Sandhya, N., Krishnan, R., \&Babu, D. R. (2020, May): A Framework for Degraded Kannada Character Recognition. In International Conference on Image Processing and Capsule Networks (pp. 735-745). Springer, Cham.

[8] Aradhya, V. M., Kumar, G. H., Noushath, S., \&Shivakumara, P. (2006): Fisher linear discriminant analysis based technique useful for efficient character recognition. In 2006 Fourth International Conference on Intelligent Sensing and Information Processing (pp. 49-52). IEEE.

[9] Saini, A., Daniel, S., Saini, S., \& Mittal, A. (2021). KannadaRes-NeXt: A Deep Residual Network for Kannada Numeral Recognition. In Machine Learning for Intelligent Multimedia Analytics (pp. 63-89). Springer, Singapore.

[10] Chandrakala, H. T., \&Thippeswamy, G. (2020): Deep Convolutional Neural Networks for Recognition of Historical Handwritten Kannada Characters. In Frontiers in Intelligent Computing: Theory and Applications (pp. 69-77). Springer, Singapore.

[11] Thippeswamy, G., \&Chandrakala, H. T. (2020): Recognition of Historical Handwritten Kannada Characters Using Local Binary Pattern Features. International Journal of Natural Computing Research (IJNCR), 9(3), 1-15.

[12] Kumar, H. S., \&Ramakrishnan, A. G. (2020). LipiGnani: A Versatile OCR for Documents in any Language Printed in Kannada Script. ACM Transactions on Asian and Low-Resource Language Information Processing (TALLIP), 19(4), 1-23.

[13] Monisha, G. S., \&Malathi, S. (2021): Effective Survey on Handwriting Character Recognition.In Computational Methods and Data Engineering (pp. 115-131).Springer, Singapore.

[14] Narayanan, V. S., \&Kasthuri, N. (2020): An efficient recognition system for preserving ancient historical documents of English characters. Journal of Ambient Intelligence and Humanized Computing, 1-9.

[15] Aravinda, C. V., Reddy, K. U. K., Meng, L., \&Prabhu, G. A. (2020, December): South Indian Character Recognition Using Statistical Feature Extraction and Distance Classifier. In 2020 International Conference on Advanced Mechatronic Systems (ICAMechS) (pp. 302-307). IEEE.

[16] Imran, F., Hossain, M. A., \& Al Mamun, M. (2020, June): Identification and Recognition of Printed Distorted Characters Using Proposed DCR Method. In 2020 IEEE Region 10 Symposium (TENSYMP) (pp. 1478-1481). IEEE.

[17] Bannigidad, P., \&Gudada, C. (2019): Age-type identification and recognition of historical kannada handwritten document images using HOG feature descriptors. In Computing, communication and signal processing (pp. 1001-1010). Springer, Singapore.

[18] Kumar, H. S., \&Ramakrishnan, A. G. (2019, February): Gamma Enhanced Binarization-An adaptive nonlinear enhancement of degraded word images for improved recognition of split characters. In 2019 National Conference on Communications (NCC) (pp. 1-6). IEEE.

[19] Ramalingam, K., \&Bhojan, R: Identification of Broken Characters in Degraded Documents, International journal of intelligent Engineering and Systems, 2017, 130-137.

[20] ParashuramBannigidad, ChandrashekarGudada: Historical Kannada Handwritten Character Recognition using K-Nearest Neighbour Technique. Journal of Artificial Intelligence Research \& Advances. 2019; 6(1): 23-29p.

[21] Gangamma, B., \&Srikanta, M. K. (2011): Enhancement of degraded historical kannada documents. International Journal of Computer Applications, 29(11), 1-6.

[22] Ranganatha, D., \& Holi, G. (2015, June): Hybrid binarization technique for degraded document images. In 2015 IEEE International Advance Computing Conference (IACC) (pp. 893-898). IEEE.

[23] Kumar, M., Jindal, M. K., \& Sharma, R. K. (2011, November): k-nearest neighbor based offline handwritten Gurmukhi character recognition. In 2011 International Conference on Image Information Processing (pp. 1-4). IEEE.

[24] Karthik, S., Mamatha, H. R., \& Murthy, K. S. (2012): An approach based on run length count for denoising the kannada characters. International Journal of Computer Applications, 50(18), 42-46. 
[25] Antony, P. J., Savitha, C. K., \&Ujwal, U. J. (2016, May):Haar features based handwritten character recognition system for Tulu script. In 2016 IEEE International Conference on Recent Trends in Electronics, Information \& Communication Technology (RTEICT) (pp. 65-68). IEEE.

[26] Sandhya, N., Krishnan, R., \&Babu, D. R. (2015, June): A novel local enhancement technique for rebuilding Broken characters in a degraded Kannada script. In 2015 IEEE International Advance Computing Conference (IACC) (pp. 176-179). IEEE.

[27] Sridevi T N, LalithaRangarajan: "Binary Image Analysis Technique for Preprocessing of Excessively dilated characters in Aged Kannada", International Journal of Recent Technology and Engineering, Volume-8 Issue-4, November 2019.

[28] https://www.newocr.com

\section{BIOGRAPHIES OF AUTHORS}

\begin{tabular}{|l|l|}
\hline & $\begin{array}{l}\text { Sridevi T.N received her Master's degree in Computer Applications(MCA) in 2005 from } \\
\text { Visvesvaraya Technological University, Belgaum. She worked as Assistant Professor in } \\
\text { Department of MCA for 13 years in the field of teaching which includes a research experience } \\
\text { of } 6 \text { years. She is currently, a research Scholar at Department of Studies in Computer Science, } \\
\text { University of Mysore, ManasaGangothri Campus, Mysuru, working under the guidance of } \\
\text { Dr.LalithaRangarajan. Her Expertise is in C and C++ software developments. Her technical } \\
\text { interests are programming and machine Automation. }\end{array}$ \\
\hline & $\begin{array}{l}\text { Dr. LalithaRangarajan has been working as a Professor in the Department of Studies in } \\
\text { Computer Science, University of Mysore, ManasaGangothri Campus, Mysuru. She has Master's } \\
\text { degree in Mathematics from Madras University, India and from School of Industrial } \\
\text { Engineering, Purdue, USA. Her career started with teaching mathematics and since 1988 shifted } \\
\text { to Computer Science. She has received her PhD degree in 2005 from the University of Mysore. } \\
\text { She has over 36 years of teaching and 21 years of research experience and contributed to the } \\
\text { field of Artificial Intelligence, Image Processing, Pattern Recognition, Cryptography, } \\
\text { Computational Biology and Bioinformatics.She has served the University at various capacities. } \\
\text { She has guided 12 Ph.D's and has about 100+ publications in peer reviewed journals and } \\
\text { proceedings of conferences to her credit. }\end{array}$ \\
\hline
\end{tabular}

